\title{
Straftäterbehandlung und Evaluation
}

\author{
Klaus-Peter Dahle ${ }^{1}$
}

Online publiziert: 25. Januar 2016

(C) Springer-Verlag Berlin Heidelberg 2016

\section{Offender treatment and evaluation}

Vor rund 25 Jahren fanden in Deutschland nach langer Zeit erstmals wieder internationale Fachtagungen (z. B. die 2nd European Conference on Law and Psychology 1990 in Nürnberg) und nationale Fachtagungen (z. B. die 4. Arbeitstagung der Fachgruppe Rechtspsychologie in der Deutschen Gesellschaft für Psychologie 1991 in Berlin) zum Schwerpunktthema Straftäterbehandlung statt. Auf diesen Tagungen glaubten die Ausrichter und Referenten nach einem Jahrzehnt weitgehender Skepsis und wissenschaftlicher Zurückhaltung Anzeichen einer Wiederbelebung des Behandlungsgedankens im deutschen Justizvollzug und einer damit einhergehenden Revitalisierung des wissenschaftlichen Interesses am Thema auszumachen. Die wissenschaftliche Debatte spaltete sich damals einerseits in eine Position, die eine Konzentration der Bemühungen auf wissenschaftlich fundierte und empirisch untermauerte Effektnachweise von sozialtherapeutischen und anderweitigen Behandlungsmaßnahmen im Sinne eines „What works?“ forderten, um auf diese Weise dem Behandlungsgedanken, wohl auch auf politischer Ebene, Nachdruck zu verleihen. Auf der anderen Seite fand sich die Gegenposition, die einer einseitigen Konzentration auf die Erforschung bloßer Globaleffekte skeptisch gegenüberstand und stattdessen eine verstärkte Beforschung von Wechselwirkungen im Sinne eines „What works how with whom under which conditions?" [1] forderte sowie für ein verstärktes Bemühen um theoretische Fundierung von Straftäterbehandlung eintrat.

Klaus-Peter Dahle

klaus-peter.dahle@charite.de

Institut für Forensische Psychiatrie, Charité -

Universitätsmedizin Berlin, Berlin, Deutschland
Es dauerte ein weiteres knappes Jahrzehnt, bis der Gedanke um Wiederbelebung des Behandlungsanliegens im Justizvollzug auch politisches Gehör und Eingang in die Gesetzgebung fand. Mit dem „Gesetz zur Bekämpfung von Sexualdelikten und anderen gefährlichen Straftaten" vom 26.01.1998 und der damit einhergegangenen Reform des § 9 StVollzG wurde dann erstmals seit dem fehlgelaufenen Versuch der Etablierung von Sozialtherapie als Maßregel der Besserung und Sicherung für eine bestimmte Gruppe von Sexualstraftätern ein sozialtherapeutischer Behandlungsanspruch formuliert. Den jährlichen Stichtagserhebungen der Kriminologischen Zentralstelle Wiesbaden e. V. zur Sozialtherapie in Deutschland zufolge haben sich seither die Zahl der sozialtherapeutischen Einrichtungen und die Zahl der dort verfügbaren Behandlungsplätze vervielfacht.

$\mathrm{Zu}$ dieser Ausweitung der Sozialtherapie in Deutschland hat ferner beigetragen, dass dezidierte sozialtherapeutische Behandlungseinrichtungen seit Mitte der 2000er Jahre auch Einzug in den Jugendstrafvollzug genommen haben. Aus neueren gesetzlichen Entwicklungen in den Bundesländern lässt sich weiterhin unschwer die Prognose ableiten, dass der Kreis der Tätergruppen mit gesetzlich verankertem sozialtherapeutischen Behandlungsanspruch in naher Zukunft wohl eher noch größer und insbesondere um Täter mit gravierenden Gewaltdelikten erweitert werden wird. Aber auch der Bundesgesetzgeber hat mit seinen jüngeren Reformen der Sicherungsverwahrung neue Aufgaben für die Sozialtherapie und für neue Behandlungseinrichtungen in Deutschland formuliert. Insbesondere der neu geschaffene $\S 66 \mathrm{c}$ StGB (Ausgestaltung der Unterbringung in der Sicherungsverwahrung und des vorhergehenden Strafvollzugs) sichert den in Sicherungsverwahrung Untergebrachten, aber auch den von Sicherungsverwahrung bedrohten Strafgefangenen, ein intensives, fachgerechtes und notfalls maßgeschneidertes Behandlungsangebot mit dem Ziel zu, 
den Vollzug dieser Maßregel zeitlich auf das Mindestmögliche zu begrenzen bzw. ganz entbehrlich zu machen. Doch nicht nur im stationären Setting von Justizvollzugsanstalten und Behandlungseinrichtungen für Sicherungsverwahrte haben therapeutische Behandlungsangebote an Gewicht gewonnen, sie haben sich auch außerhalb von Vollzugseinrichtungen verbreitet. So sind in den vergangenen Jahren nahezu flächendeckend in Deutschland forensisch-therapeutische Nachsorgeambulanzen entstanden, deren Aufgabe es u. a. ist, ehemalige Gefangene mit gravierenden Delikten und erhöhtem Rückfallrisiko die ersten Jahre nach dem Freiheitsentzug auf ihrem Rückweg in die Gesellschaft therapeutisch zu begleiten.

Insoweit scheint es, dass der Behandlungsgedanke in den vergangenen rund 15 Jahren tatsächlich in Deutschland eine Renaissance erfahren hat. Die Entwicklungen stellen die Behandlungseinrichtungen vor Herausforderungen, da nicht nur neue Aufgaben zu bewältigen sind, sondern sich die Einrichtungen auch zunehmend juristisch erstrittenen Behandlungsansprüchen und juristischen Rechtfertigungserfordernissen im Hinblick auf die Qualität des Behandlungsangebots gegenübersehen und sich nicht mehr nur auf therapeutische Behandlungsindikationen zurückziehen können. Mit dem neuen $\S 66 \mathrm{c} \mathrm{StGB}$ ist es darüber hinaus absehbar, dass - sollte die Absicht des Gesetzgebers erfolgreich sein und sollten tatsächlich zukünftig die therapeutisch erreichbaren sicherungsverwahrten und von Sicherungsverwahrung bedrohten Gefangenen verstärkt entlassen werden können - in den Einrichtungen eine zunehmend schwierige Klientel verbleiben wird, die bislang als nicht oder zumindest als nur sehr schwer mit therapeutischen Mitteln ansprechbar galt. Dies wird neue Behandlungskonzepte erfordern, aber auch sehr geduldige, kreative, gut ausgebildete und frustrationstolerante Behandler.

Die Gesetzesentwicklungen der vergangenen Jahre haben aber nicht nur durch die Ausweitung der Aufgabenstellungen für Behandlungseinrichtungen die Bedeutung von Straftäterbehandlung innerhalb und außerhalb des Justizvollzugs gestärkt. Die neueren Vollzugsgesetze der Bundesländer sehen zudem weitgehend flächendeckend die Evaluation von Maßnahmen der Straftäterbehandlung im Hinblick auf ihre Wirksamkeit vor. Dementsprechend mehren sich Aktivitäten, die sich um die Umsetzung dieses gesetzlichen Auftrags bemühen, wenngleich die Länder hier derzeit unterschiedliche Wege beschreiten. So werden in einigen Bundesländern Evaluationen durch die Justizbehörden selbst, namentlich durch die kriminologischen Dienste der Länder durchgeführt, während andere Bundesländer stärker auf Evaluationen durch externe Wissenschaftseinrichtungen setzen. Unabhängig davon stehen die Evaluatoren ihrerseits vor neuen Herausforderungen, da gesetzlich fixierte Behandlungsansprüche klassischen (gematchten oder gar randomisierten) Vergleichsgruppenuntersuchungen aus behandelten und unbehandelten Paarlingen entgegenstehen und wachsende postmurale Behandlungsangebote und Präventionsbemühungen bei entlassenen Tätern mit erhöhtem Rückfallrisiko die Identifikation etwaiger Behandlungseffekte und deren Rückführung auf die unterschiedlichen stattgefundene Maßnahmen zusätzlich erschweren oder gar verunmöglichen. Globale Wirksamkeitsuntersuchungen im Sinne der eingangs erwähnten Frage nach dem „What works?" sind unter diesen Voraussetzungen kaum mehr umsetzbar.

Die Lösung kann letztlich nur darin bestehen, Evaluationen stärker auf die Analyse von Wechselwirkungen im Sinne der Frage nach dem „What works how, with whom under which conditions?" zu konzentrieren. Es geht also mehr darum, unterschiedliche Rahmenbedingungen und Organisationsformen von Behandlungseinrichtungen, unterschiedliche Behandlungsbedarfe und -voraussetzungen aufseiten ihrer jeweiligen Klientel, aber auch Qualifikationen, Teamfaktoren und Leitungsstrukturen aufseiten der Einrichtung sowie unterschiedliche Behandlungsmethoden und ihre jeweilige theoretische Fundierung im Hinblick auf ihre Wechselwirkungen vergleichend $\mathrm{zu}$ analysieren. In Anbetracht der Vielzahl potenziell relevanter Einflüsse, aber auch angesichts der eingeschränkten Variabilität einzelner Einflussfaktoren innerhalb eines Bundeslandes oder gar innerhalb einer Einrichtung, werden Einzelstudien mit dieser Aufgabe überfordert sein. Sie können allenfalls Ausschnitte möglicher Wechselwirkungen in den Blick nehmen und müssen andere potenzielle Einflussfaktoren mangels Varianz, zu geringer Fallzahl oder fehlender Ressourcen ausblenden. Um diese Einschränkung längerfristig zu überwinden, bedarf es übergreifender Analysen, die auf einen zunehmend großen Datenpool zurückgreifen und die Ergebnisse von Einzelevaluationen zusammenführen können. Um ein solches Anliegen in Zukunft realisieren zu können, bedarf es aber der Einigung auf gewisse Mindeststandards für Evaluationen von Behandlungseinrichtungen und -maßnahmen im Justizvollzug, die zunächst sicherstellen, dass die verschiedenen Untersuchungen von ihrer Stoßrichtung her vergleichbare Ziele verfolgen. Es bedarf weiterhin der Definition von Schnittstellen, Bewertungsmaßstäben und eines Pools von Erhebungsmethoden und -instrumenten, um zu gewährleisten, dass die verschiedenen Datensätze miteinander kompatibel sind und zueinander in Beziehung gesetzt werden können. Insoweit wäre eine bundesweite Task Force, die solche Standards entwickelt, begrüßenswert und angesichts des aktuell steigenden Evaluationsbedarfs auch geboten. Dies erfordert aber auch den politischen Willen der Bundesländer, solche landesübergreifenden Initiativen zu fördern und bundesweite Auswertungen von Einzelevaluationen zu ermöglichen. Derzeit hat es den Anschein, dass mancherorts mit der Veröffentlichung von 
Evaluationsergebnissen und der Zugänglichmachung der zugehörigen Daten noch eher zögerlich umgegangen wird.

Die skizzierten aktuellen Entwicklungen und die damit einhergehenden potenziellen Chancen für die Weiterentwicklung fundierter Behandlungsangebote im Justizvollzug sind der Grund dafür, sich mit dem vorliegenden Schwerpunktheft der Zeitschrift Forensische Psychiatrie, Psychologie, Kriminologie dem Thema zu widmen. Dabei werden einige Einzelevaluationen zu speziellen Behandlungsangeboten aus verschiedenen Einrichtungen vorgestellt (Beiträge von Elsner und König, von Gouzoulis-Mayfrank sowie von Voß et al.), der Beitrag von Suhling und Guéridon beschreibt hingegen einen vergleichenden methodenund institutionsübergreifenden Ansatz zur Evaluation der Sozialtherapie in Niedersachsen. Der Beitrag von Endres und Breuer widmet sich schließlich dem grundsätzlichen Problem des methodischen Umgangs mit Abbrechern im Rahmen von Evaluationsstudien.

Klaus-Peter Dahle

Interessenkonflikt. K.-P. Dahle gibt an, dass kein Interessenkonflikt besteht.

\section{Literatur}

1. Steller M (1994) Behandlung und Behandlungsforschung. In: Steller M, Dahle K-P, Basqué M (Hrsg) Straftäterbehandlung. Centaurus, Pfaffenweiler, S 3-12 